\title{
Relationships between aging of PAHs and soil properties
}

\author{
Lei Luo $^{\mathrm{a}, \mathrm{c}}$, Shu Lin ${ }^{\mathrm{a}, \mathrm{b}}$, Honglin Huang ${ }^{\mathrm{a}}$, Shuzhen Zhang ${ }^{\mathrm{a}, *}$ \\ a State Key Laboratory of Environmental Chemistry and Ecotoxicology, Research Center for Eco-Environmental Sciences, Chinese Academy of Sciences, Beijing 100085, China \\ ${ }^{\mathrm{b}}$ TUV Rheinland Mandy Co., Ltd, Fuzhou 350013, China \\ ${ }^{\mathrm{c}}$ Department of Geosciences, Princeton University, Princeton, NJ 08544, United States
}

\section{A R T I C L E I N F O}

\section{Article history:}

Received 7 February 2012

Received in revised form

4 May 2012

Accepted 1 July 2012

\section{Keywords:}

Polycyclic aromatic hydrocarbon

Aging

Hard organic carbon

Porosity

Diffusion

\begin{abstract}
A B S T R A C T
Sequestration and diffusion of three polycyclic aromatic hydrocarbons (PAHs) in seven Chinese soils were investigated for up to 200 days in sterile soil microcosms as functions of soil property and aging time. The aging of the PAHs, assessed using a mild extractant that removes primarily the labile fraction, showed a biphasic behavior. The rapid diffusion from labile to nonlabile domains was mainly dependent upon the distribution of meso- and micropore fraction and total organic carbon content. Meanwhile, the slow diffusion was found to decrease with the increase of the content of soil organic carbon, particularly of hard organic carbon $(p<0.01)$ and the meso- and micropore fraction, as well as with the increasing molecular size of PAHs. This work offers evidence that analyses of organic carbon fractionation and porosity are important to adequately assess the mechanistic basis of sequestration and diffusion of organic contaminants in soils.
\end{abstract}

(c) 2012 Elsevier Ltd. All rights reserved.

\section{Introduction}

Aging of hydrophobic organic contaminants (HOCs) is an important attenuation process which often entails an initially rapid and reversible sorption process followed by a period of slow diffusion occurring over weeks, months, or even years once they released into the environment (Alexander, 2000). Extensive literature has confirmed that extractability and bioavailability of HOCs decreased significantly with time in aging processes (Northcott and Jones, 2001; Ju and Young, 2005; Birdwell et al., 2007), which can be significantly rate-limiting for in situ biodegradation, remediation, and subsurface transport, resulting in overestimation of exposure and risk from these compounds (Alexander, 2000; Ginsbach et al., 2010). Thus, a better understanding of the diffusion processes and the relevant mechanism of HOCs during aging is critical for predicting their environmental fate and developing strategies for remediation of polluted soils.

Recent investigations have proposed that physicochemical properties of organic carbon (OC) are predominantly responsible for the sequestration of HOCs in soils (Tao et al., 2006; Luo et al., 2008). More specifically, Ju and Young (2005) suggested that the labile (rapidly desorbing) fraction of HOCs was inversely related to the hard or recalcitrant OC fraction in soils. Some investigations

\footnotetext{
* Corresponding author.

E-mail address: szzhang@rcees.ac.cn (S. Zhang).
}

demonstrated that dissolved organic carbon (DOC) can exert a positive effect on the extractability of polycyclic aromatic hydrocarbons (PAHs) in soils (Tao et al., 2006; Luo et al., 2008). On the other hand, Abu and Smith (2006) indicated that the nonlabile fraction of phenanthrene in soils was retained within narrow width mesopores and micropores. In addition, clay can also exert an appreciable effect on PAH sequestration in soils (Luo et al., 2008). Aging processes of HOCs are closely related to their sorption and sequestration in soils. However, a comprehensive understanding on the effects of soil properties on the long-term sequestration of HOCs such as PAHs is still lacking. Meanwhile, knowledge on roles of the different fractions of $\mathrm{OC}$ in the aging processes of HOCs is also very limited (Ginsbach et al., 2010).

Previous experimental evidence indicates that aging of HOCs, as a time-dependent process, can be successfully described with multirate first-order diffusion models (Cornelissen et al., 1998; Johnson et al., 2001; Birdwell et al., 2007). Much work has been attempted to construct the relationships between theoretical diffusion rate constants and functionalities including contaminant properties and sorbent properties to accurately predict and thus remediate the environmental contamination. For example, Ginsbach et al. (2010) observed that rapid release rates were governed by the OC contents in natural geosorbents. While Birdwell et al. (2007) suggested that more than one factor may contribute to the sequestration of HOCs in soils based on multiple studies from different sorption history and conditions. Therefore, it is posited that more extensive investigations based on multicomponent 
properties, for example, the fractions of OC with different rigidities and the distribution of meso- and micropores, would provide more accurate information on the relationship between HOC aging processes and soil properties, bringing helpful insights into the relevant mechanisms of HOC aging.

The objectives of this study were to relate soil properties to the sequestration and diffusion of typical HOCs including phenanthrene (Phe), pyrene (Pye), and benzo[a]pyrene $(\mathrm{B}[\mathrm{a}] \mathrm{P})$, and to explore key factors affecting the PAH aging to establish the quantitative relationship between diffusion rate constants and soil properties. A series of soils with varying OC and clay contents were used. A mild extractant of $n$-butanol was applied to reflect the extractability and diffusion processes of the PAHs in soils, which has been demonstrated to be a successful predictor for the bioavailability of PAHs in soils (Tang and Alexander, 1999; Northcott and Jones, 2001). A unique feature of this study is the comprehensive assessment of the effects of soil properties, particularly of OC fractions and pore distribution, on the sequestration and diffusion of PAHs in a series of aged soils with different periods up to 200 days.

\section{Materials and methods}

\subsection{Materials}

Phenanthrene, pyrene, and benzo[a]pyrene for soil spiking were obtained from Acros Organics Co. (>97\%). Other solvents used including $n$-hexane, acetone, methanol, and $n$-butanol ( $\mathrm{BuOH}$ ) were of pesticide grade. All glassware was cleaned in an ultrasonic cleaner and heated at $350{ }^{\circ} \mathrm{C}$ for $12 \mathrm{~h}$.

Seven surface $(0-20 \mathrm{~cm})$ soils with different $O C$ contents were collected from various locations in China (Fig. S1 in the Supporting Information, SI). Soil samples were air-dried, homogenized and ground to pass through a $0.5 \mathrm{~mm}$ sieve, and then sterilized by gamma irradiation $(2.5 \mathrm{Mrad})$ from a ${ }^{60} \mathrm{Co}$ source for subsequent experiments.

Soil samples were artificially spiked with the three PAHs using a fully validated and described procedure (Northcott and Jones, 2001; Tao et al., 2006). Briefly, $10 \mathrm{~mL}$ of hexane solution and $2.5 \mathrm{~mL}$ of stock solution $(1.0 \mathrm{~g} / \mathrm{L}$ of each PAH compound in $n$ hexane) were added to a blender, to which $250 \mathrm{~g}$ of air-dried soil samples were added as five additions with $50 \mathrm{~g}$ each time and blended for $20 \mathrm{~s}$ between the additions and finally for a full $5 \mathrm{~min}$. The nominal concentration of each individual $\mathrm{PAH}$ compound was $10 \mathrm{mg} / \mathrm{kg}$. The spiked soil samples were stored in an open glass container under a sterile fume hood for $24 \mathrm{~h}$ until all hexane evaporated and subsequently aged in the amber jars sealed with depolished glass lids as the sterile microcosms in the dark at room temperature. The volume of the jars is about $250 \mathrm{~mL}$ and little headspace volume was left when the spiked soils were stored. The sterility of the microcosms was tested using standard microbiological techniques.

\subsection{Soil characterization}

Soils were analyzed for $\mathrm{pH}$, OC content, particle size distribution, and specific surface area and porosity. Soil $\mathrm{pH}$ was measured at a soil to water ratio of 1:5 (w/v). Particle size distribution was obtained by Laser Particle Analyzer (Malvern Mastersizer 2000) after destroying the aggregates in soils by sodium hexametaphosphate (Jiang et al., 2005). Specific surface area and pore size distribution from 1.7 to
$300 \mathrm{~nm}$ were determined by applying the multipoint BET model to adsorption and desorption isotherms of $\mathrm{N}_{2}$ gas at $77 \mathrm{~K}$ with an ASAP 2020 accelerated surface area and porosimetry analyzer (Micromeritics Co.). In order to evaluate the effects of characteristics of heterogeneous OC on the aging of the PAHs in the soils, soil OC was characterized in terms of soft (including DOC) and hard OC, and black carbon (BC), respectively, which was conducted as described below. Contents of Phe, Pye, and B [a]P in the pristine soils were below the detection limit.

Hard OC was quantified after wet oxidation (with $\mathrm{K}_{2} \mathrm{~S}_{2} \mathrm{O}_{8}$ to OC at a ratio of 12:1) at $120^{\circ} \mathrm{C}$ for $4 \mathrm{~h}$ to remove the easily oxidized OC (Li and Werth, 2001; Ju and Young, 2004). Total OC (TOC) was measured after acid treatment $(10 \% \mathrm{HCl})$ to remove inorganic carbon. Carbon contents in the treated samples and the pristine soils were measured with an elemental analyzer (PE2400, Perkin Elmer Inc.). Soft OC fraction was calculated based on difference between TOC and hard OC. Among various hard OC in soils, BC was selected to be quantified according to the methods of Gelinas et al. (2001). Briefly, soil samples were firstly demineralized with a mixture of $\mathrm{HCl}$ and $\mathrm{HF}$ acids to expose any biochemical entrapped in a mineral matrix. The hydrolysable $\mathrm{OC}$ fraction was then removed with $\mathrm{O}_{2}$-free trifluoroacetic acid and $\mathrm{HCl}$, after which the non- $\mathrm{BC} \mathrm{OC}$ fraction was finally removed by thermal oxidation at $375{ }^{\circ} \mathrm{C}$ for $24 \mathrm{~h}$. Contents of DOC, extracted with $0.01 \mathrm{~mol} / \mathrm{L} \mathrm{NaCl}$ for $6 \mathrm{~h}$, were determined using a Phoenix 8000 TOC analyzer (Malvern Co.). Selected physicochemical properties of the soils are listed in Table 1 .

\subsection{Soil extraction procedures}

The aging processes of the PAHs in the soils were evaluated by sampling the PAH spiked soils at predetermined time intervals during the 200-day aging period. Replicate samples from the soil microcosms were subjected to $\mathrm{BuOH}$ shake extraction using the procedure summarized as below. Each soil sample (1.0 g) was extracted with $10 \mathrm{~mL} \mathrm{BuOH}$ in a PTFE-lined cap glass centrifuge tube of $45 \mathrm{~mL}$ capacity on a flat bed shaker for $24 \mathrm{~h}$. Our preliminary experiment suggested that an extracting time of $24 \mathrm{~h}$ produced more precise results for the extractable PAHs in soils compared with $12 \mathrm{~h}$ (Northcott and Jones, 2001). An aliquot of $1.0 \mathrm{~mL}$ of the supernatant was transferred to a $\mathrm{KB}$ flask after the extracts were separated by centrifugation at $4000 \times \mathrm{g}$ for $20 \mathrm{~min}$. The $\mathrm{BuOH}$ in the extracts was evaporated by blowing nitrogen. Then the extracts were dissolved in $1.0 \mathrm{~mL}$ methanol, which was subjected to PAH analyses. Analyses were conducted using an Agilent $1200 \mathrm{HPLC}$ and a Kromasil Eternity C18 column $(4.6 \times 250 \mathrm{~mm}, 5 \mu \mathrm{m})$ as described previously (Luo et al., 2008).

\subsection{Model calculation}

The aging processes of the PAHs in soils were described with a biphasic firstorder model, which describes diffusion rates for both the rapid diffusion fraction, $\Phi_{\text {rap, }}$, and the slow diffusion fraction, $\Phi_{\text {slow }}=\left(1-\Phi_{\text {rap }}\right.$ ) (Cornelissen et al., 1997; Johnson et al., 2001; Birdwell et al., 2007):

$$
q_{t} / q_{0}=\Phi_{\text {rap }} \exp \left(-k_{\text {rap }} t\right)+\left(1-\Phi_{\text {rap }}\right) \exp \left(-k_{\text {slow }} t\right)
$$

where $q_{t}$ and $q_{0}$ are the BuOH-extractable amounts of PAHs after time $t$ (day) and at the start of the aging experiment, respectively; and $k_{\text {rap }}$ and $k_{\text {slow }}$ are the rate constants of rapid and slow diffusion, respectively. $\Phi_{\text {rap }}, k_{\text {rap }}$ and $k_{\text {slow }}$ were determined by exponential curve fitting using Origin 7.5 program (OriginLab Corp.), and fits were carried out by minimizing the squares of the differences between experimental and calculated values of $q_{t} / q_{0}$.

To better understand the unit effects of soil properties on the diffusion processes as a whole system, optimal multiple linear regression (MLR) models were constructed to predict the key factors affecting the aging processes. More detailed MLR model information was provided in the SI.

Table 1

Selected physicochemical properties of the soils studied.

\begin{tabular}{|c|c|c|c|c|c|c|c|c|c|c|}
\hline \multirow[t]{2}{*}{ Location } & \multirow[t]{2}{*}{ Order $^{\mathrm{a}}$} & \multirow[t]{2}{*}{$\mathrm{pH}$} & \multirow{2}{*}{$\begin{array}{l}\text { DOC } \\
(\mathrm{mg} / \mathrm{kg})\end{array}$} & \multicolumn{4}{|c|}{ Organic carbon (\%) } & \multirow[t]{2}{*}{ Clay (\%) } & \multirow{2}{*}{$\begin{array}{l}\text { BET-N } 2 \text { SA } \\
\left(\mathrm{m}^{2} / \mathrm{g}\right)^{\mathrm{d}}\end{array}$} & \multirow{2}{*}{$\begin{array}{l}\text { Average pore } \\
\text { diameter }(\mathrm{nm})\end{array}$} \\
\hline & & & & Total & Soft $^{b}$ & Hard & $\mathrm{BC}^{\mathrm{C}}$ & & & \\
\hline Beijing & Alfisol & 7.2 & 438 & 1.27 & 0.36 & 0.91 & 0.23 & 19.9 & 40.5 & 5.08 \\
\hline Gansu & Aridisol & 7.9 & 370 & 0.84 & 0.41 & 0.43 & 0.15 & 19.6 & 47.8 & 8.98 \\
\hline Guangdong & Ultisol & 6.1 & 200 & 1.18 & 0.10 & 1.08 & 0.08 & 29.3 & 84.1 & 6.51 \\
\hline Hainan & Oxisol & 4.9 & 253 & 0.96 & 0.04 & 0.92 & 0.12 & 66.1 & 147.3 & 5.72 \\
\hline Heilongjiang & Mollisol & 6.3 & 851 & 2.82 & 0.57 & 2.25 & 0.07 & 40.4 & 61.1 & 4.99 \\
\hline Inner Mongolia & Mollisol & 7.9 & 243 & 1.98 & 0.44 & 1.54 & 0.08 & 37.1 & 79.8 & 5.45 \\
\hline Shaanxi & Aridisol & 7.7 & 278 & 0.64 & 0.21 & 0.43 & 0.06 & 27.5 & 64.2 & 5.42 \\
\hline
\end{tabular}

a Orders are based on the United States Department of Agriculture soil classification system.

b Soft OC is calculated as the difference between the TOC and hard OC contents.

c BC, black carbon, is determined by thermal oxidation at $375{ }^{\circ} \mathrm{C}$ method (Gelinas et al., 2001).

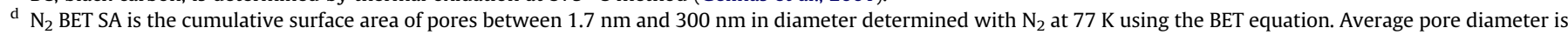
estimated from pore volume $(V)$, and surface area $(A)$ : diameter $=4 \mathrm{~V} / \mathrm{A}$. 


\subsection{Quality evaluation and statistical analysis}

The concentrations of PAHs were measured prior to (unspiked soil), immediately after the spiking experiments and at the end of the aging stage by dichloromethane Soxhlet extraction. Recovery of the three PAHs from the freshly spiked soils (aged for 0 day) ranged from $90.2 \pm 5.2 \%$ to $107.6 \pm 1.7 \%$ for Phe, and $91.3 \pm 4.3 \%$ to $108.1 \pm 5.8 \%$ for Pye and $98.3 \pm 6.4 \%$ to $103.1 \pm 2.3 \%$ for $\mathrm{B}[\mathrm{a}] \mathrm{P}$, indicating the spiking procedures were validated. While recovery of the PAHs from the aged soils (for 200 days) by Soxhlet extraction ranged from $77.0 \pm 3.1 \%$ to $84.1 \pm 5.2 \%$ for Phe, and $78.6 \pm 4.3 \%$ to $83.8 \pm 3.4 \%$ for Pye and $75.3 \pm 1.4 \%$ to $81.9 \pm 2.3 \%$ for B[a]P. All samples were analyzed in duplicate in the study.

Statistical analyses of the results were performed using software SPSS 17.0 (SPSS Inc.). Linear regression analyses were used to establish a relationship among variables with $p<0.05$. Prediction models for the key controlling parameters under certain conditions were developed by MLR using a forward-stepwise procedure at a 0.05 significance level.

\section{Results and discussion}

\subsection{Aging processes of PAHs in soils}

The quantities of PAHs extracted from the soils with $\mathrm{BuOH}$, described as the percentage extracted, decreased quickly at the beginning and then gradually leveled off with increasing aging time as shown in Fig. 1. The biphasic first-order model can well fit the aging processes of PAHs in the soils, with correlation coefficients $(R)$ larger than 0.972 (Table S1). From the location of the bends in the curves, it is clear that the labile fraction of PAHs declined rapidly after 15-30 days of aging. Particularly, the extractable amounts of PAHs in the soils decreased over 20\% after 3 days compared with their initial spiking levels. After a rapid decreasing period, the extractable fraction of the PAHs experienced a slow but stable decrease with time. Based on the biphasic first-order model, the values of $k_{\text {rap }}$, ranging from 0.135 to 0.390 days $^{-1}$, were about 150-1000 times larger than those of $k_{\text {slow, which corresponded }}$ with the clear distinction between fast and slow fractions in the PAH aging curves (Fig. 1). Compared with previous results, the values of both the $k_{\text {rap }}$ and $k_{\text {slow }}$ showed a lower degree of variation among the tested soils while the $k_{\text {slow }}$ values were a little lower than those of the published data obtained for the similar aging periods (200 days vs 250 days) (Johnson et al., 2001), probably because of different extractants (BuOH vs Tenax) adopted and OC contents $(0.64-2.82 \%$ here vs $0.5-8.3 \%)$ in the soils in the experiments (Johnson et al., 2001). However, the ratios between $k_{\text {rap }}$ and $k_{\text {slow }}$ were comparable to the reported results, validating the biphasic behavior of organic compounds in aged soils. In addition, the extractability of the PAHs in the soils decreased generally with increasing compound molecular weight (Fig. 1).

It is necessary to note that the extractability of the PAHs by Soxhlet extraction, significantly higher than that of $\mathrm{BuOH}$ extraction $(p<0.01)$, also decreased from their initial levels of nearly $100 \%$ to about $80 \%$ over the 200 days of aging time, which was comparable to the published data obtained from the sealed sterile microcosms with similar aging periods (Macleod and Semple, 2000; Johnson et al., 2002). After 240-day incubation, Johnson et al. (2002) observed that $61-70 \%$ of PAHs were extracted by Soxhlet extraction, while Macleod and Semple (2000) found about 16-20\% of Pyr could not be extracted with a sequential extraction scheme including Soxhlet extraction after aged for 168 days based on a radiolabelled experiment. The results validated the reliability of the microcosm sealing and maintenance of sterile conditions in our experiments, and thus the possible decrease in the extractability from biodegradation or volatilization was generally minimized. Therefore, although it appears impossible to totally avoid the influence of the loss of PAHs from soil on accurately describing their aging processes, the influence levels were thought acceptable.

\subsection{Effects of soil properties on extractability of PAHs in aged soils}

Changes in the amounts of extractable PAHs with time reflect their aging processes in the soils. Therefore, correlation analysis was initially conducted between the extractable PAHs and the key soil properties. As expected, the extractable amounts of individual PAHs at given times (here by taking the aging times of 30,107 and 200 days as the examples) negatively correlated with total OC contents (with $p<0.05$ for Phe, and $p<0.01$ for Pyr and B[a]P, respectively), and also a weak negative relationship existed between the extractable amounts of PAHs and clay contents (Fig. 2).
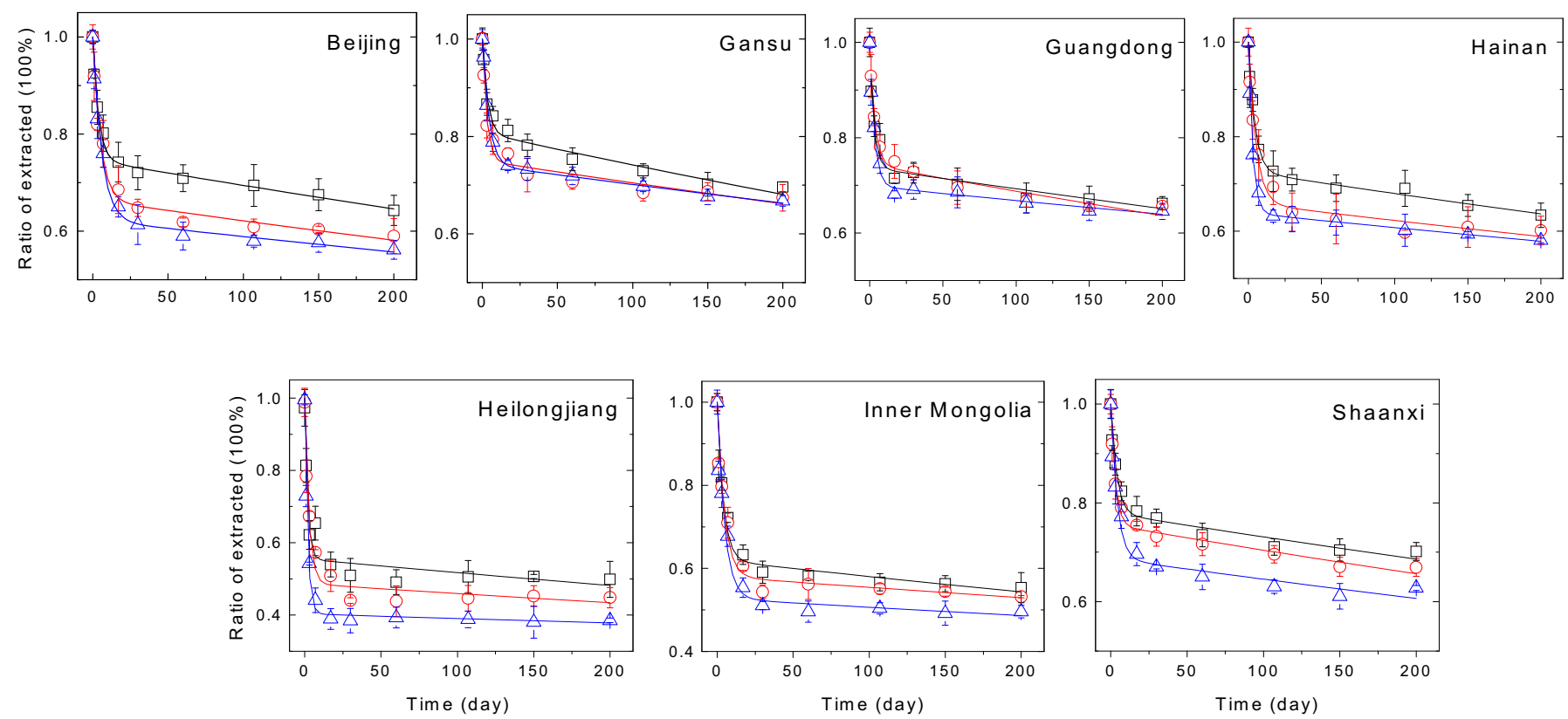

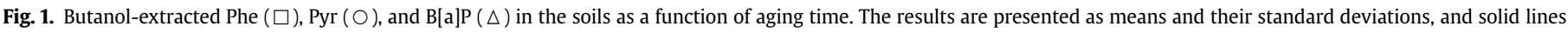
are obtained by exponential curve fitting. 

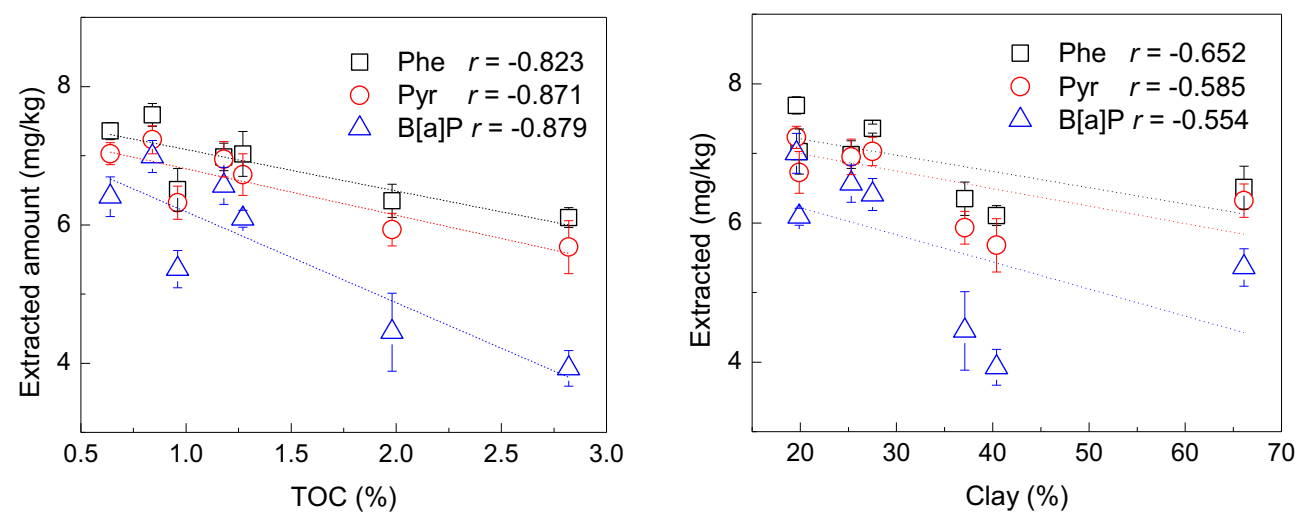

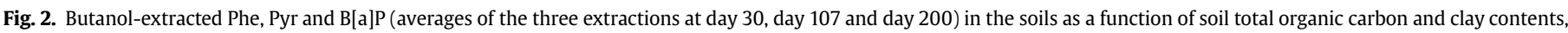
respectively. The results are presented as means and their standard deviations.

No obvious link between the extractable PAHs and soft OC (including DOC) or BC was observed in the soils.

The influence of various OC fractions on PAH sequestration has been suggested by previous investigations (Luo et al., 2008, 2010). According to the well accepted dual reactive domain model (Xing and Pignatello, 1997), hard OC (or glassy OC) exhibits a competitive and hysteretic sorption as the main sorption domains for $\mathrm{HOC}$ and predominantly controlled the sequestration of HOCs in soils. With the increase of the hard OC contents, PAHs exhibited a strong tendency to the sorption domains. Consequently, the extractable fraction of PAHs, particularly of the larger molecular PAH with higher hydrophobicity, in the soils significantly decreased (Fig. 2). On the contrary, the fraction of PAHs adsorbed by soft OC can be relatively easy to be released by the polar extractant and thus no significant relationship was observed between the extractable PAHs and the soft OC contents. However, it is surprising that no relationship between the extractable PAHs and recalcitrant $\mathrm{BC}$ content was discernable although a variety of investigations have indicated that BC have a strong affinity for PAHs (Abu and Smith, 2006; Agarwal and Bucheli, 2011). This phenomenon could be ascribed to the fact that $\mathrm{BC}$ content accounted for only about $10 \%$ of the TOC content, much lower compared with the data reported in the previous studies (Xiao et al., 2004). Moreover, the strong sorption domain (i.e. the micropore system) of the tiny $\mathrm{BC}$ fraction in the soils might be blocked or occupied by soil components such as DOC and mineral clays, thus attenuating the possible influence from BC (Kwon and Pignatello, 2005; Brändli et al., 2008; Xiao et al., 2012). Agarwal and Bucheli (2011) also observed the only governor of TOC in the distribution of PAHs in the organic carbon-rich agricultural soils.

\subsection{Effects of soil properties on diffusion of PAHs in aged soils}

It is well accepted that the decrease in extractability is attributed to the sorption of PAHs to the organic fractions and/or soil minerals, and/or diffusion of the PAHs into the micropores within the solid phases of soils (Northcott and Jones, 2001; Johnson et al., 2002). As stated above, the aging processes of the PAHs, indicated by the gradually decrease in their extractability over time, can be accurately described with two rate constants $\left(k_{\text {rap }}\right.$ and $\left.k_{\text {slow }}\right)$ in the two distinct stages, respectively (Johnson et al., 2001). Correlation analysis revealed that $k_{\text {rap }}$ was negatively dependent on TOC to a certain extent while no significance level was obtained. The pore distribution also exerted an important effect on the diffusion of HOCs (Pignatello and Xing, 1996; Farrell et al., 1999; Abu and Smith, 2006). Particularly, it was proposed that the pore fraction with diameters below $6 \mathrm{~nm}$ (hereafter specifically called $\mathrm{PF}_{6 \mathrm{~nm}}$ for short) predominately controlled the sorption of HOCs (Farrell and Reinhard, 1994; Pignatello and Xing, 1996). If the $\mathrm{PF}_{6} \mathrm{~nm}$, for example, was applied to correlate with the $k_{\text {rap }}$, a negative relationship could be observed (Fig. S2 in SI). Furthermore, the comparable pore diameter (about 4 times larger than the PAH molecular size) was believed to be sensitive to HOC sequestration and diffusion in geomaterials (Farrell et al., 1999; Abu and Smith, 2006). In addition, a large portion of the cumulative pore volumes (about 35-65\%) in the soils came from the pores with diameters smaller than $6 \mathrm{~nm}$ as shown in Fig. 3. On the other hand, a significant inverse relationship was obtained between $k_{\text {slow }}$ and TOC ( $p<0.05$, with $r$ values of $0.812,0.798$ and 0.776 for Phe, Pyr and $\mathrm{B}[\mathrm{a}] \mathrm{P}$, respectively) and more significant correlation occurred if hard OC was adopted instead $(p<0.01)$ (Fig. 4). In addition, a negative correlation was observed between $k_{\text {slow }}$ and $\mathrm{PF}_{6 \mathrm{~nm}}$ (with $r$ values of $0.744(p<0.05), 0.660(p<0.1)$ and $0.626(p<0.1)$ for Phe, Pyr and $\mathrm{B}[\mathrm{a}] \mathrm{P}$, respectively) while no distinct relationship was identified between $k_{\text {slow }}$ and soil clay content.

Once released into soils, PAHs can rapidly access the sorption domains via meso- and micropore diffusion (Fig. S3 in SI) (Pignatello and Xing, 1996; Farrell et al., 1999; Abu and Smith, 2006). Considering the PAH molecular sizes (larger than $1.1 \mathrm{~nm}$, Table S1 in SI), it is reasonable to presume that the narrow mesoand micropores comparable to the molecular sizes of PAHs are the limiting factors for the rapid diffusion processes (Nam and

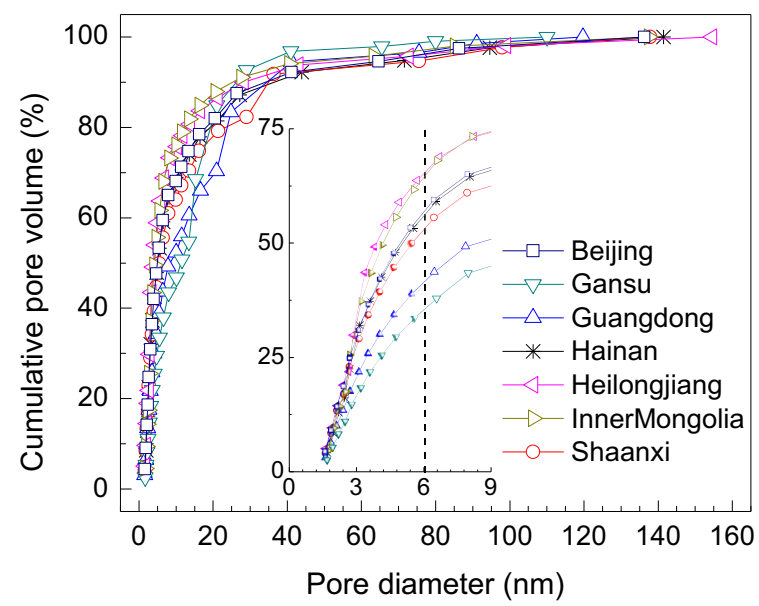

Fig. 3. Cumulative pore volume distribution as a function of pore diameter in the soils. The inset figure shows the cumulative pore volume distribution in a shorter scale up to $9 \mathrm{~nm}$. 


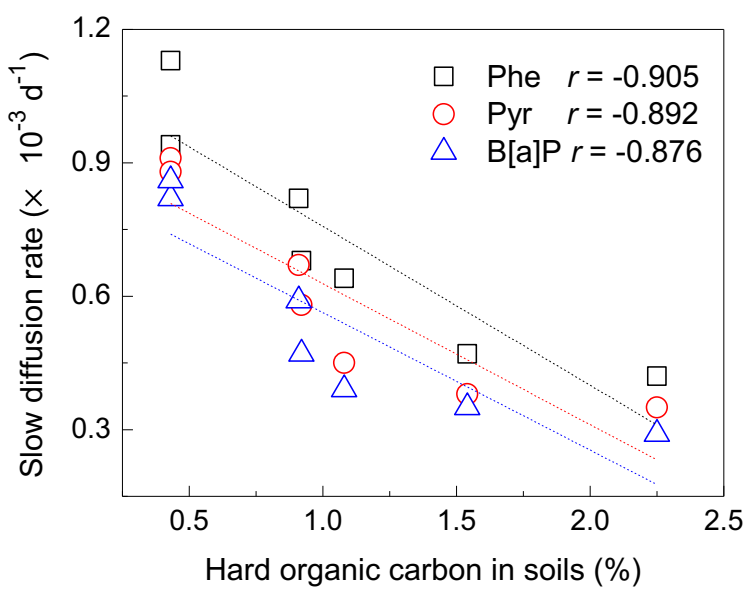

Fig. 4. Slow diffusion rate constants of Phe, Pyr and $\mathrm{B}[\mathrm{a}] \mathrm{P}$ (averages of the three extractions at day 30, day 107 and day 200) in the soils as a function of soil hard organic carbon content. The results are presented as the fitted values and their standard deviations were not shown due to large deviations (Table S1 in SI).

Alexander, 1998; Abu and Smith, 2006). Actually, with the increase of the hydrophobic meso- and micropores in soils, the extractability tended to decrease (with $r$ values of $0.686(p<0.1)$, $0.760(p<0.05)$ and $0.789(p<0.05)$ for Phe, Pyr and B[a]P, respectively) (Fig. S4 in SI). The negative relationship between $\mathrm{PF}_{6} \mathrm{~nm}$ and $k_{\text {rap }}$ verified that the meso- and micropore distribution was one of the limiting factors for the rapid diffusion of PAHs in soils. With the increase of $\mathrm{PF}_{6} \mathrm{~nm}$ in soils, the diffusion of PAHs was consequently retarded. The evidence that no appreciable relationship existed between $k_{\text {rap }}$ and the whole pore distribution (reflected by the average pore diameter and the total surface area) further indicated the important contribution of the $\mathrm{PF}_{6} \mathrm{~nm}$ to the rapid diffusion of PAHs in soils. Meanwhile, it was expected that TOC and clay can exert a negative influence on the sequestration and then the diffusion of a wide range of HOCs (Northcott and Jones, 2001; Tao et al., 2006; Luo et al., 2008). For example, Ginsbach et al. (2010) suggested that TOC was negatively correlated with the $k_{\text {rap }}$ of HOCs. However, no significant correlation between the $k_{\text {rap }}$ and TOC or clay content was obtained in the soils. The heterogeneity of soil matrices allows for many variables to contribute to the diffusion of PAHs in soils, and if not predominant, the effect of a single variable could be attenuated by the influences from other variables.

After a rapid diffusion in the meso- and micropores, the PAHs suffered a slow, progressive diffusion. It was proposed that slow diffusion could be attributed to the diffusion through meso- and micropores (Pignatello and Xing, 1996; Nam and Alexander, 1998; Abu and Smith, 2006) and/or diffusion into the internal micropores of OCs or clay (Huang and Weber, 1997; Northcott and Jones, 2001; Bogan and Trbovic, 2003) in geomaterials. Condensed OC present can impact the distribution of hydrophobic micropores in soils (Ju and Young, 2004; Kwon and Pignatello, 2005). During the diffusion processes, the PAHs were preferentially bound to the condensed OC domains and recalcitrant to mild extractants in soils (Tao et al., 2006). As a result, with the increase of hard OC in the soils, the slow diffusion rates were greatly limited by the strong affinity of PAHs in the condensed domain (Ju and Young, 2004) and exhibited a negative relationship with the hard OC in the soils (Fig. 4). The negative correlation between $k_{\text {slow }}$ and $\mathrm{PF}_{6} \mathrm{~nm}$ also verified the limit of $\mathrm{PF}_{6} \mathrm{~nm}$ to slow diffusion from the condensed $\mathrm{OC}$ to a certain extent. For the different $\mathrm{PAH}$ compounds, their diffusion rates consequentially tended to be slower with the increase of their molecular sizes and hydrophobicity (Fig. 4 and Fig. S2 in SI).

\subsection{Quantification the relationship between PAH aging and soil properties}

Considering the multi-contributions of various soil properties, it is expectable that MLR models can accurately quantify the diffusion processes of PAHs in aged soils. Based on the MLR analysis (Table S3 in SI), the best regression models describing the relationship between the diffusion rate constants ( $k_{\text {rap }}$ and $\left.k_{\text {slow }}\right)$ and the soil properties were obtained as follows:

$$
\begin{aligned}
k_{\text {rap }}= & -0.456\left[\mathrm{PF}_{6 \mathrm{~nm}}\right]-0.003[\mathrm{TOC}] \\
& +0.436 \quad\left(R^{2}=0.793, \alpha<0.05\right) \\
k_{\text {slow }}= & -3.3^{*} 10^{-4}[\text { hard OC }]-4.7^{*} 10^{-6}\left[\mathrm{PF}_{6 \mathrm{~nm}}\right] \\
& +7.1^{*} 10^{-5} \quad\left(R^{2}=0.923, \alpha<0.05\right)
\end{aligned}
$$

Eqs (2) and (3) verified that the diffusion of aged PAHs could be well quantified by some key factors including TOC/hard OC and $\mathrm{PF}_{6} \mathrm{~nm}$. Specifically, $\mathrm{PF}_{6 \mathrm{~nm}}$ was the first independent variable fitted to the rapid diffusion process and could explain $75.0 \%$ of the variability in the $k_{\text {rap }}$. It is necessary to note that only $79.3 \%$ of the variability in the $k_{\text {rap }}$ can be explained even when the contributions of both porosity and TOC were considered, indicating there are still some other properties that could influence the rapid process and thus examining more soil samples is necessary to accurately characterize the labile diffusion process.

For the slow diffusion process, only hard OC content and $\mathrm{PF}_{6} \mathrm{~nm}$ were selected as the predictors (explanatory variables) and including clay content as a predictor improved the model slightly as shown in Table S3 in SI, indicating that the contribution of hard $\mathrm{OC}$ and $\mathrm{PF}_{6} \mathrm{~nm}$ was much greater than that of clay under both conditions. It is noteworthy that the hard $O C$ alone can explain over $81 \%$ of variability in the $k_{\text {slow }}$ (Table S3 in SI). Given that the effects of OC and porosity on the diffusion of PAHs in soils, it is safe to presume that hard $\mathrm{OC}$ and $\mathrm{PF}_{6} \mathrm{~nm}$ can successfully be used to predict the PAH aging since they can explain over $92 \%$ of variability in the $k_{\text {slow }}$ (Table S3 in SI). It is necessary to note that the values of $k_{\text {rap }}$ and $k_{\text {slow }}$ used here were the average of the three PAHs since the compounds exhibited similar aging trend in the soils.

\subsection{Environmental implications}

Our results suggest that the aging processes of PAHs in soils are controlled by several key factors including OC compositions and structures, and pore distribution. As described in Fig. S3 (in SI), once released into soils, PAHs might be initially adsorbed onto outer humic surfaces or larger mesopores of geomaterials, and then diffuse with time into previously inaccessible pores within the soil carbonaceous matrix (Nam and Alexander, 1998; Abu and Smith, 2006). Hard organic carbon and porosity distribution can act as better predictors of PAH sequestration in soils than TOC and surface area, respectively. Findings of this work should also help understand the underlying sorption mechanisms of HOCs by OC in geomaterials and predict the multimedia transport and bioavailability of HOCs in aged contaminated sites.

\section{Acknowledgments}

We thank Dr. Rebecca L. Sanders in Princeton University for her assistance in manuscript writing. Financial support for this work was provided by the National Natural Science Foundation of China (Grant No. 41023005, 20890110 and 20907064). 


\section{Appendix A. Supplementary material}

Supplementary material associated with this article can be found, in the online version, at http://dx.doi.org/10.1016/j.envpol. 2012.07.003.

\section{References}

Abu, B., Smith, S., 2006. Mechanistic characterization of adsorption and slow desorption of phenanthrene aged in soils. Environmental Science and Technology 40, 5409-5414.

Agarwal, T., Bucheli, T.D., 2011. Is black carbon a better predictor of polycyclic aromatic hydrocarbon distribution in soils than total organic carbon? Environmental Pollution 159, 64-70.

Alexander, M., 2000. Aging, bioavailability, and overestimation of risk from environmental pollutants. Environmental Science and Technology 34, 4259-4265.

Birdwell, J., Cook, R.L., Thibodeaux, L.J., 2007. Desorption kinetics of hydrophobic organic chemicals from sediment to water: a review of data and models. Environmental Toxicology and Chemistry 26, 424-434.

Bogan, B.W., Trbovic, V., 2003. Effect of sequestration on PAH degradability with Fenton's reagent: roles of total organic carbon, humin, and soil porosity. Journal of Hazardous Materials 100, 285-300.

Brändli, R.C., Hartnik, T., Henriken, T., Cornelissen, G., 2008. Sorption of native polyaromatic hydrocarbons (PAH) to black carbon and amended activated carbon in soil. Chemosphere 73, 1805-1810.

Cornelissen, G., van Noor, P.C.M., Parsons, J.R., Govers, H.A.J., 1997. Temperature dependence of slow adsorption and desorption kinetics of organic compounds in sediments. Environmental Science and Technology 31, 454-460.

Cornelissen, G., Rigterink, H., Ferdinandy, M.M.A., van Noor, P.C.M., 1998. Rapidly desorbing fractions of PAHs in contaminated sediments as a predictor of the extent of bioremediation. Environmental Science and Technology 32, 966-970.

Farrell, J., Reinhard, M., 1994. Desorption of halogenated organics from model solids, sediments, and soil under unsaturated conditions. 2. Kinetics. Environmental Science and Technology 28, 63-72.

Farrell, J., Grassian, D., Jones, M., 1999. Investigation of mechanisms contributing to slow desorption of hydrophobic organic compounds from mineral solids. Environmental Science and Technology 33, 1237-1243.

Gelinas, Y., Prentice, K.M., Baldock, J.A., Hedges, J.I., 2001. An improved thermal oxidation method for the qualification of soot/graphitic black carbon in sediment and soils. Environmental Science and Technology 35, 3519-3525.

Ginsbach, J.W., Killops, K.L., Olsen, R.M., Peterson, B., Dunnivant, F.M., 2010. Fraction of organic carbon predicts labile desorption rates of chlorinated organic pollutants in laboratory-spiked geosorbents. Environmental Toxicology and Chemistry 29, 1049-1055.

Huang, W., Weber Jr., W.J., 1997. A distributed reactivity model for sorption by soils and sediments. 10. Relationships between desorption, hysteresis, and the chemical characteristics of organic domains. Environmental Science and Technology 31, 2562-2569.

Jiang, W., Zhang, S.Z., Shan, X.Q., Feng, M.H., Zhu, Y.G., McLaren, R.G., 2005. Adsorption of arsenate on soils. Part 1: laboratory batch experiments using 16 Chinese soils with different physicochemical properties. Environmental Pollution 138, 278-284.
Johnson, M.D., Keinath II, T.M., Weber Jr., W.J., 2001. A distributed reactivity mode for sorption by soils and sediments. 14. Characterization and modeling of phenanthrene desorption rates. Environmental Science and Technology 35, 1688-1695.

Johnson, D.L., Jones, K.C., Langdon, C.J., Piearce, T.G., Semple, K.T., 2002. Temporal changes in earthworm availability and extractability of polycyclic aromatic hydrocarbons in soil. Soil Biology and Biochemistry 34, 1363-1370.

Ju, D., Young, T.M., 2004. Effects of competitor and natural organic matter characteristics on the equilibrium sorption of 1,2-Dichlorobenzene in soil and shale. Environmental Science and Technology 38, 5863-5870.

Ju, D., Young, T.M., 2005. The influence of natural organic matter rigidity on the sorption, desorption, and competitive displacement rates of 1,2-dichlorobenzene. Environmental Science and Technology 39, 7956-7963.

Kwon, S., Pignatello, J.J., 2005. Effect of natural organic substances on the surface and adsorptive properties of environmental black carbon (char): pseudo pore blockage by model lipid components and its implications for $\mathrm{N}_{2}$-probed surface properties of natural sorbents. Environmental Science and Technology 39 7932-7939.

Li, J., Werth, C.J., 2001. Evaluating competitive sorption mechanisms of volatile organic compounds in soils and sediments using polymers and zeolites. Environmental Science and Technology 35, 568-574.

Luo, L., Zhang, S.Z., Ma, Y.B., 2008. Evaluation of impacts of soil fractions on phenanthrene sorption. Chemosphere 2008 (72), 891-896.

Luo, L., Zhang, S.Z., Christie, P., 2010. New insights into the influence of heavy metals on phenanthrene sorption in soils. Environmental Science and Technology 44 7846-7851.

Macleod, C.J.A., Semple, K.T., 2000. Influence of contact time on extractability and degradation of pyrene in soils. Environmental Science and Technology 34, 4952-4957.

Nam, K., Alexander, M., 1998. Role of nanoporosity and hydrophobicity in sequestration and bioavailability: tests with model solids. Environmental Science and Technology 32, 71-74.

Northcott, G.L., Jones, K.C., 2001. Partitioning, extractability, and formation of nonextractable $\mathrm{PAH}$ residues in soil. 1. Compound differences in aging and sequestration. Environmental Science and Technology 35, 1103-1110.

Pignatello, J.J., Xing, B.S., 1996. Mechanisms of slow sorption of organic chemicals to natural particles. Environmental Science and Technology 30,1-11.

Tang, J.X., Alexander, M., 1999. Mild extractability and bioavailability of polycyclic aromatic hydrocarbons in soil. Environmental Toxicology and Chemistry 18 2711-2714.

Tao, S., Xu, F.L., Liu, W.X., Cui, Y.H. Coveney Jr. R.M., 2006. A chemical extraction method for mimicking bioavailability of polycyclic aromatic hydrocarbons to wheat grown in soils containing various amounts of organic matter. Environmental Science and Technology 40, 2219-2224.

Xiao, X.Y., Li, F.L., Huang, J.X., Sheng, G.D., Qiu, Y.P., 2012. Reduced adsorption of propanil to black carbon: effect of dissolved organic matter loading mode and molecule size. Environmental Toxicology and Chemistry. http://dx.doi.org $10.1002 /$ etc. 1800

Xiao, B.H., Yu, Z.Q., Huang, W.L., Song, J.Z., Peng, P.A., 2004. Black carbon and kerogen in soilsand sediments. 2. Their roles in equilibrium sorption of lesspolar organic pollutants. Environmental Science and Technology 38, 5842-5852.

Xing, B.S., Pignatello, J.J., 1997. Dual-mode sorption of low-polarity compounds in glassy poly (vinyl chloride) and soil organic matter. Environmental Science and Technology 31, 792-799. 